\title{
CONDICIONALIDAD, SELECTIVIDAD Y AFECTACIÓN: ¿ESTRATEGIAS A CONSIDERAR PARA LA RECUPERACIÓN ECONÓMICA?
}

\author{
María Amparo Grau Ruiz \\ Catedrática de Derecho Financiero y Tributario \\ Universidad Complutense de Madrid (España) \\ Directora de la Revista Técnica Tributaria
}

En el actual entorno de fragilidad, patente en muchas de las estructuras que sostienen la vida económica -como secuela de la urgencia en las actuaciones para atajar la crisis sanitaria provocada por la reciente pandemia-, corresponde ahora a todos quienes, de alguna manera, cuentan con la posibilidad de apoyar la recuperación del bienestar social, trabajar en la misma dirección que antes han marcado al unísono los profesionales del mundo de la salud ante el brutal impacto causado en nuestra sociedad. Todos a una, con las herramientas de que podamos disponer, en el intento de solventar el problema inminente y buscar a la vez nuevas soluciones útiles a medio $\mathrm{y}$ largo plazo ${ }^{1}$.
Las rápidas reacciones en el campo tributario -como en el laboral y de protección social-son cruciales ante tamaña tempestad y afortunadamente no se han hecho esperar. Los entes públicos han adoptado una variedad de medidas de distinto calado, según su ámbito competencial, para tratar de hacer frente a los nefastos efectos del coronavirus. Su mayor o menor éxito se podrá juzgar en el futuro ${ }^{2}$. Lo que ya resulta innegable es que esta crisis ha servido de catalizador de cambios inmediatos, y en esta tesitura hace falta redirigir algunas de las primeras respuestas y acomodar otras adicionales para afrontar, en el mejor modo posible, la siguiente etapa en esta improvisada carrera por salir adelante.

\footnotetext{
1 El Banco de España ha hecho públicas el 8 de junio de 2020 las Proyecciones macroeconómicas de la economía española (2020-2022): contribución del Banco de España al ejercicio conjunto de proyecciones del Eurosistema de junio de 2020. Nota informativa disponible en https://www.bde.es/ f/webbde/GAP/Secciones/SalaPrensa/NotasInformativas/Briefing_notes/es/notabe080620.pdf [última consulta 10 de junio de 2020].

2 El Tribunal de Cuentas Europeo: «Los auditores examinarán la respuesta de la economía y la sanidad pública ante el brote de COVID-19 en la UE», Comunicado de prensa, Luxemburgo 28 de mayo de 2020. Disponible en https://www.eca.europa.eu/es/Pages/NewsItem.aspx?nid=13821 [última consulta 10 de junio de 2020].
} 
La política económica depende, en gran medida, de la política fiscal y, en ésta, importa el qué y cómo se ponga en marcha. El panorama presente está, en general, caracterizado por la pérdida de poder adquisitivo, el aumento de las bolsas de pobreza y la aversión al riesgo. En esta situación límite, parece haberse incrementado el nivel de exposición de las instituciones públicas a la crítica sobre la correcta utilización del gasto público y su financiación.

La presión sobre las arcas públicas es creciente, para atender las demandas de socorro que formulan personas y empresas en posición de vulnerabilidad. En consecuencia, las Administraciones tributarias, sienten la necesidad de forzar la movilización sus recursos internos. Y aquí surge el problema: ¿Movilizar se reduce a aumentar la recaudación tributaria? Esto puede hacerse de diferentes formas: ¿expandir la base fiscal?, ¿subir los tipos?, ¿recortar beneficios fiscales?, ¿condicionarlos y controlarlos?

En esta coyuntura, una subida de impuestos puede llegar a ser contraproducente. La desconfianza y el «por si acaso» pueden ahuyentar a quienes están en posición de contribuir por su capacidad económica o crear valor en nuestro territorio. Nadie ha dudado de la necesidad de inyectar liquidez en la primera fase de lucha contra esta crisis, o de la conveniencia de aplazamientos, moratorias legales y sectoriales, o de la procedencia de los avales del ICO. Toca ahora generar confianza en los mercados y que la inversión de los fondos públicos sea productiva, de manera que las empresas puedan crear riqueza para favorecer la recuperación económica, atendiendo al clamor popular; posibilitar que con el ahorro

precautorio en algunos casos pronto se vuelva a invertir y consumir, para estar en condiciones de poder nutrir regularmente al Fisco combatiendo el déficit en las cuentas públicas.

En este contexto, cobra especial interés la condicionalidad, puesta de manifiesto ya sea en las ayudas directas o en las indirectas (a través de beneficios fiscales), en tanto permite claramente fomentar la buena gobernanza extra-financiera con el propósito de generar determinados impactos positivos a los que es -más que nuncaespecialmente sensible la sociedad española. Más allá de las subvenciones, el crédito o la deuda, ¿por qué no apostar por más apoyo público (directo o indirecto) a las empresas, condicionado a conductas socialmente responsables? Es más, es posible no renunciar al avance hacia los Objetivos de Desarrollo Sostenible, adaptando la senda de su concreto cumplimiento por parte de nuestro Estado, en función de las necesidades más acuciantes derivadas de la pandemia.

Al revisar las líneas de acción propuestas por algunas organizaciones internacionales y diferentes países; en el fondo, late esta tendencia común - perfectamente comprensible- a aumentar la dosis de condicionalidad en las ayudas, acompañada de la selectividad al desnudo, sin reparos de algunos Estados a la hora de otorgar la ayuda deseada cuando se está en disposición de hacerlo, y que hace mirar a muchos con nuevos ojos a ciertas figuras tributarias, que podrían gravar a quienes han salido beneficiados económicamente por/ tras la crisis, postulando la afectación de la potencial recaudación, incluso 
debatiéndose ya a qué nivel territorial se asignarían pues a veces hasta se incluyen en distintas cestas de tributos ${ }^{3,4}$.

La condicionalidad salta así al primer plano en muchas de las estrategias que se proponen para garantizar el correcto uso de los limitados recursos disponibles en estas circunstancias tan extremadamente delicadas. Además, esta forma excepcional de proceder suele admitirse con carácter temporal. En principio, el régimen comunitario de ayudas de Estado se habría flexibilizado hasta que se produjera la recuperación económica. El problema entonces se traslada de sede al término ${ }^{5}$. Mientras tanto, la Comisión Europea consciente de los riesgos que entrañan determinados comportamientos para el mercado único, propone instrumentos para

3 «Los países van a necesitar ingentes recursos públicos. Ello va a agudizar la búsqueda de nuevos viveros recaudatorios y de nueva materia imponible, lo que generará debates sobre los taxmix nacionales. [...] si se debe acelerar la poda de beneficios fiscales en el impuesto de sociedades para mejorar la capacidad de recaudación del impuesto, haciendo compatible la presión fiscal sobre las empresas con la protección de su capacidad para generar riqueza y empleo. [...] Y, por supuesto, debería adaptarse el régimen de ayudas de Estado ante el escenario que se abre en la implantación de políticas económicas en relación con la crisis del coronavirus». César García Novoa, «El día de mañana de la fiscalidad. Los cambios tributarios en el escenario post Covid-19», Taxlandia, 19 de nayo de 2020. https://www.politicafiscal.es/cesar-garcia-novoa/el-dia-de-mananade-la-fiscalidad-los-cambios-tributarios-en-el-escenario-post-covid-19 [última consulta 10 de junio de 2020]

4 Los nuevos recursos propios complementarían los recursos propios tradicionales, un recurso propio basado en el IVA simplificado y las contribuciones nacionales, así como nuevos recursos propios basados en los residuos de envases de plástico no reciclados. [...] Las opciones podrían incluir un recurso propio basado en el régimen de comercio de derechos de emisión, incluida su posible ampliación a los sectores marítimo y de la aviación, y un mecanismo de ajuste en frontera de las emisiones de carbono. [...] Las empresas que obtienen enormes beneficios del mercado único de la UE y que sobrevivirán la crisis, también gracias a la ayuda directa e indirecta tanto de la UE como nacional, podrían contribuir a la reconstrucción del mismo en la fase de recuperación. Así pues, cabría contemplar un recurso propio basado en operaciones de las empresas que, en función de su diseño, podría producir alrededor de 10000 millones EUR anuales. Un impuesto digital se basaría en los trabajos de la OCDE sobre la fiscalidad de las empresas con una presencia digital significativa. En este sentido, la Comisión apoya activamente los debates dirigidos por la OCDE y el G20 y está dispuesta a actuar si no se alcanza un acuerdo global. Un impuesto digital aplicado a las empresas con un volumen de negocios superior a los 750 millones EUR podría generar hasta 1300 millones EUR anuales para el presupuesto de la UE». COMUNICACIÓN DE LA COMISIÓN AL PARLAMENTO EUROPEO, AL CONSEJO EUROPEO, AL CONSEJO, AL COMITÉ ECONÓMICO Y SOCIAL EUROPEO Y AL COMITÉ DE LAS REGIONES El presupuesto de la UE: motor del plan de recuperación para Europa, COM/2020/442 final, Bruselas, 27 de mayo de 2020, pp. 16-17. Disponible en https://eur-lex.europa.eu/ legal-content/ES/TXT/?qid=1591759410119\&uri=CELEX:52020DC0442 [última consulta 10 de junio de 2020].

5 Sobre la posible conflictividad que puede acarrearse en caso de la adecuada falta de definición en las ayudas, baste esta ilustrativa la referencia: «En un período como el que estamos viviendo, en que el gasto público se dispara, y deberá mantenerse al alza por más tiempo, sin promesa de ingresos públicos cuantiosos, se debe ser especialmente escrupuloso en la concesión de ayudas y evitar disposiciones que permitan, bien que haya quien las aproveche de manera injustificada, bien que puedan ser interpretadas fundadamente en diversas formas y su implementación genere, en el medio plazo, una ingente cantidad de reclamaciones». Rosa Fraile Fernández, «El concepto jurídico indeterminado que marcará el devenir de los gastos públicos relativos a los nuevos ERTE», Blog de la Red de Profesores de Derecho Financiero y Tributario, 19 de mayo de 2020. https://rpdft.org/ 2020/05/19/el-concepto-juridico-indeterminado-que-marcara-el-devenir-de-los-gastos-publicosrelativos-a-los-nuevos-erte-rosa-fraile-fernandez/ [última consulta 10 de junio de 2020] 
mejorar la solvencia económica de los más débiles ${ }^{6}$.

Se ha de tratar de aprovechar bien este momento en el que cabe la diferenciación financiera y fiscal a escala estatal para enderezar nuestra economía mediante la articulación de una red de apoyos inteligentes (en su caso, vía incentivos). Dado que no va a faltar la financiación condicionada a una transformación justa, digital y verde, apóyese fiscalmente el desempeño sostenible de las PYMES; ábranse vías sencillas para regularizar, de manera inclusiva, la situación de quienes viven dependiendo de un empleo informal en la economía sumergida; impúlsese la próxima incorporación de unos 300.000 jóvenes al mercado laboral o complétese su formación en las habilidades necesarias para la transición justa, de forma que podamos evitar otra generación perdida tras la triste experiencia con la anterior crisis financiera; poténciense los servicios ecosistémicos que la Naturaleza aporta gratis, pero que muchos mantienen en lastimosas condiciones precarias en el ámbito rural; cuídense la I+D+IT y la eficiencia energética, etc. La clave para encaminar acertadamente la elección de medidas hacia el bien común parece residir en la búsqueda de un enfoque transversal, que promueva el efecto multiplicador, con el ajuste de los debidos límites.

La creatividad fruto de la colaboración público-privada es imprescindible para marcar la orientación de las reformas. No hay que perder la esperanza en el entendimiento de todos los actores implicados, si bien estos deben evitar, al fijar las condiciones, la aparición una maraña de compatibilidades o no (como en el caso de la renta mínima). En ningún caso deberían exigirse condiciones que pudieran impedir la supervivencia de las PYMES, procurando apoyar siempre la continuidad empresarial. Convendría idear un sistema de condicionalidad dinámico ${ }^{7}$. Cualquier ventaja fiscal habría de vincularse a condiciones realistas, fáciles de aplicar gradualmente en diferentes estadios, según la posible comprobación (con información suficientemente detallada disponible) del desempeño eficaz.

6 «En algunos Estados miembros, el Estado estará en condiciones de intervenir en apoyo de estas empresas, mientras que, en otros, la capacidad de apoyo público será más limitada. Los efectos económicos de la pandemia de COVID-19 también han sido desiguales en los Estados miembros. Si no se toman medidas, estas diferencias provocarán una distorsión permanente de las condiciones de competencia y agravarán las divergencias económicas dentro de la Unión. El instrumento de apoyo a la solvencia contrarrestará estas distorsiones y contribuirá al buen funcionamiento del mercado único. [...] El instrumento complementaría las políticas nacionales que apenas unos pocos Estados miembros están introduciendo y garantizaría la disponibilidad de apoyo a la solvencia en toda la Unión. Las condiciones de los instrumentos deben ser coherentes con las normas sobre ayudas estatales para garantizar la igualdad de condiciones de competencia y facilitar posibles combinaciones con las ayudas directamente facilitadas por los Estados miembros, además de atender, como corresponde, a la naturaleza europea del instrumento y a la gestión comercial de los fondos». COM/2020/442 final, p. 8 .

7 En evolución a la luz de los acontecimientos, al estilo de los prestamos del Fondo Monetario Internacional, haciendo un parangón entre el sector empresarial y los países. «Cuando un país obtiene crédito del FMI, el gobierno se compromete a ajustar la política económica para superar los 
En la actualidad, en el marco de la ONU, se han contabilizado 5.400 zonas económicas especiales en 147 economías. La mayoría ofrece incentivos fiscales, junto con otras ventajas. Están surgiendo algunas centradas en nuevas industrias como la alta tecnología, más allá de las tradicionales. También hay margen para construir nuevas zonas y atraer inversión en actividades relevantes desde el punto de vista de los Objetivos de Desarrollo Sostenible. Por ejemplo, expresamente se reconoce que los incentivos fiscales se pueden condicionar no sólo al empleo, la inversión o la exportación, sino también a indicadores sociales y medioambientales ${ }^{8}$.

Conviene tener presente, en cualquier caso, la clara postura de la Comisión Europea recientemente publicada:

«Unos sistemas tributarios equitativos y favorables al crecimiento podrían ayudar a respaldar la recuperación económica contribuyendo a un crecimiento inclusivo y sostenible. Unos sistemas tributarios justos y propicios al crecimiento pueden potenciar la inversión privada y mejorar el entorno empresarial, fomentar el empleo, reducir las desigualdades y contribuir a una economía resiliente desde el punto de vista medioambiental. Por otra parte, una simplificación del sistema tributario puede contribuir a limitar las distorsiones económicas y reducir las cargas administrativas para las empresas. Dado que un sistema tributario eficiente y equitativo es particularmente importante para respaldar una recuperación económica eficaz a medio plazo, a algunos Estados miembros se les recomienda que reformen sus sistemas tributarios, particularmente pasando de una fiscalidad del trabajo a una fiscalidad medioambiental.

La lucha contra la planificación fiscal agresiva sigue siendo una clara prioridad, particularmente a fin de permitir a los Estados miembros disponer de un volumen justo de ingresos tributarios para realizar su apoyo presupuestario. La transposición de la legislación de la UE y de las iniciativas acordadas a nivel internacional ayudará a reducir las prácticas de planificación fiscal agresiva. Sin embargo, algunas características de los sistemas fiscales de algunos Estados miembros

\footnotetext{
problemas que le llevaron a solicitar asistencia financiera a la comunidad internacional. Las condiciones de estos préstamos también sirven para garantizar que el país será capaz de reembolsar los recursos al FMI, de manera que esos recursos puedan ponerse a disposición de otros países miembros que los necesiten. Las reformas de los préstamos aprobadas en 2009 simplificaron la condicionalidad del FMI para ayudar a que los países se identifiquen mejor con políticas sólidas y eficaces». FMI: La condicionalidad del FMI, Ficha técnica, 6 de marzo de 2018. Disponible en https:// www.imf.org/es/About/Factsheets/Sheets/2016/08/02/21/28/IMF-Conditionality IMF, «2018 review of programme design and conditionality,» IMF Policy Paper (May 2019). Disponible en https:// www.imf.org/en/Publications/ Policy-Papers/Issues/2019/05/20/2018-Review-of-Program-Designand-Conditionality-46910 [última consulta 10 de junio de 2020].

8 UNCTAD, World Investment Report 2019: Special Economic Zones (United Nations publication, Sales No. E.19.II.D.12). ESCAP, «Foreign Direct Investment and Sustainable Development in International Investment Governance», Studies in Trade, Investment and Innovation, No. 90 (2019) Foreign Direct Investment and Sustainable Development in International Investment Governance (United Nations publication, Sales No. E.20. II.F.6, 2019). ONU, Inter-agency Task Force on Financing for Development, Financing for Sustainable Development Report 2020, Naciones Unidas, Nueva York, 2020, p. 66. Disponible en https://developmentfinance.un.org/sites/developmentfinance.un.org/ files/FSDR_2020.pdf [última consulta 10 de junio de 2020].
} 
(a saber, Chipre, Hungría, Irlanda, Luxemburgo, Malta y los Países Bajos) son utilizadas por las empresas que practican una planificación fiscal agresiva. A la luz de esas consideraciones, se recomienda a estos Estados miembros frenar la planificación fiscal agresiva» 9 .

En definitiva, se nos muestran desde instancias europeas las señas de un camino de auto-fijación de objetivos por país, de cara a afrontar las reformas pertinentes para la recuperación. Hace falta una visión de la economía que se desea para el futuro, aplicar una condicionalidad refinada, en un calendario preciso y con sistemas de seguimiento. Confiemos en que nuestros representantes encuentren proyectos comunes de corte transversal, que se preocupen de cuestiones con impacto multisectorial como la sanidad o la educación, y que pueda mejorarse la cohesión social con las herramientas fiscales.

Para optimizar el diseño de planes coordinados de recuperación, previamente cada uno tendrá que auto-evaluar su capacidad de aportar y en qué condiciones, evitando el tremendo coste de la indefinición a través del diálogo central, autonómico y local con el sector privado, y formulando propuestas fiscales coherentes para renovar el pacto social.

9 COMUNICACIÓN DE LA COMISIÓN AL PARLAMENTO EUROPEO, AL CONSEJO EUROPEO, AL CONSEJO, AL BANCO CENTRAL EUROPEO, AL COMITÉ ECONÓMICO Y SOCIAL EUROPEO, AL COMITÉ DE LAS REGIONES Y AL BANCO EUROPEO DE INVERSIONES Semestre Europeo de 2020: Recomendaciones específicas por país. COM/2020/500 final, Bruselas, 20 de mayo de 2020, pp.14-15. También reviste interés en la página 9 lo siguiente: «Los elementos presupuestarios de las recomendaciones específicas por país reflejan la activación de la cláusula general de salvaguardia, que recomienda tomar todas las medidas necesarias para combatir eficazmente la pandemia, sostener la economía y respaldar la posterior recuperación. Cuando las condiciones económicas lo permitan, las políticas fiscales deberán estar encaminadas a lograr situaciones fiscales prudentes a medio plazo y garantizar la sostenibilidad de la deuda, al mismo tiempo que se fomenta la inversión. [...] Los Estados miembros también deberán prestar atención a la calidad de las finanzas públicas al haberse adoptado paquetes de medidas fiscales sin precedentes, estableciendo de forma adecuada las prioridades para las inversiones y los gastos productivos. Por otra parte, con los paquetes de medidas fiscales sin precedentes adoptados por los Estados miembros y el consiguiente aumento del papel del sector público en la economía, es muy importante que las administraciones públicas sean eficaces y eficientes. Con vistas al futuro, los Estados miembros que logren una gestión sólida de las finanzas públicas saldrán antes de la crisis. La inversión pública y el gasto público son importantes para respaldar la transición ecológica y digital, particularmente a través de una contratación pública que tenga en cuenta los aspectos ecológicos y digitales y de una presupuestación que tenga en cuenta los aspectos ecológicos». Disponible en https://eur-lex.europa.eu/legal-content/ES/TXT/?qid=1591761923020\&uri=CELEX:52020DC0500 [última consulta 10 de junio de 2020]. 